\title{
Considerações metodológicas na interpretação do rastreamento sorológico da hepatite $B$ em doadores de sangue*
}

\author{
Celina Maria Turchi Martelli**, Ana Lúcia Sampaio Sgambatti de Andrade**, Divina das Dores \\ Paula Cardoso***, Simonne Almeida e Silva ${ }^{* * *}$, Fabio Zicker ${ }^{* *}$
}

\begin{abstract}
MARTELLI, C. M. T. et al. Considerações metodológicas na interpretação do rastreamento sorológico da hepatite B em doadores de sangue. Rev. Saúde públ., S. Paulo, 25: 11 - 6, 1991. Trata-se de parte de uma pesquisa sobre a soroprevalência e fatores de risco para as doenças infecciosas triadas pelos bancos de sangue, com o objetivo de discutir as implicações metodológicas na interpretação dos estudos seccionais realizados em bancos de sangue para estimativa da prevalência populacional para a infecção pelo Virus da Hepatite B (VHB). De outubro de 1988 a fevereiro de 1989, 1.033 primodoadores de sangue, de 5 dos 8 bancos de sangue da cidade de Goiânia - GO, Brasil, foram entrevistados. Uma amostra de sangue foi coletada para deteç̧ão dos marcadores sorológicos $\mathrm{AgHBs}$ e anti-HBs pela técnica de ELISA. Foram obtidas taxas de soroprevalência de $1,9 \%$ e $10,9 \%$ para $\mathrm{AgHBs}$ e anti-HBs, respectivamente. Não houve diferença estatísticamente significante na prevalência de $\mathrm{AgHBs}$ em diferentes grupos etários e sexo. Foi observado $\circ$ aumento significante de anticorpos anti-HBs com a idade $\left(X^{2}\right.$ para tendência $=7,9 p=0$, 004). O valor preditivo positivo e a sensibilidade da história de icterícia ou hepatite na anamnese em detectar soropositivos mostraram-se baixos, $13,6 \%$ e $2,2 \%$, respectivamente. Foram discutidas a validade interna e externa e as limitações na extrapolação destas estimativas levando-se em consideração as características de distribuição etária e sexo da população, a "voluntariedade", um possível "efeito doador saudável" e a representatividade dos grupos de risco para infecção pelo VHB entre os doadores de sangue.
\end{abstract}

Descritores Hepatite B. Sorodiagnóstico. Estudos de prevalência. Doadores de sangue.

\section{Introdução}

A prevalência da infecção pelo vírus da Hepatite B (VHB) tem sido descrita através do rastreamento sorológico de grupos populacionais selecionados como doadores de sangue ${ }^{10}$, gestantes ${ }^{12}$, grupos de risco, incluindo homossexuais masculinos $^{37,41}$, hemodialisados ${ }^{17}$, usuários de drogas endovenosas ${ }^{11}$, presidiários ${ }^{14}$, profissionais da área de saúde ${ }^{15,36} \mathrm{e}$ militares ${ }^{25}$. A primeira vista, - rastreamento sorológico de doadores de sangue parece ser uma situação privilegiada para estudos de prevalência da infecção pelo VHB, pois grande contingente de indivíduos são submetidos a triagem laboratorial de rotina, consti-

* Trabalho financiado pelo Conselho Nacional de Desenvolvimento Científico e Tecnológico (CNPq . Processo 404230-1) e Secretaria de Ciência e Tecnologia do Ministério da Saúde (Convênio 001/89 MS/UFG).

* Departamento de Saúde Coletiva do Instituto de Patologia Tropical e Saúde Pública/Universidade Federal de Goiás - Praça Universitária, s/n - Setor Universitário 74000 - Goiânia, GO - Brasil.

*** Departamento de Microbiologia do Instituto de Patologia Tropical e Saúde Pública/Universidade Federal de Goiás.

**** Bolsista do Conselho Nacional de Desenvolvimento Científico e Tecnológico ( $\mathrm{CNPq}$ ) tuindo-se em grupo populacional de fácil acesso e a baixo custo operacional. Entetanto, a interpretação dos dados provenientes de banco de sangue deve levar em consideração as características da população rastreada e os testes laboratoriais empregados.

Mesmo assim, estimativas de prevalência de infecção pelo VHB obtidas em bancos de sangue têm se constituído em referência freqüente do nivel endêmico em diferentes regiōes ${ }^{9,23,33}$.

Em trabalho anterior ${ }^{32}$ comparamos a prevalência de infecção e de fatores de risco para infecção pelo VHB em uma população de prisioneiros, considerada de alto risco, e em uma população de doadores de sangue. Para explicar a alta prevalência obtida em prisioneiros foram analisadas as diferenças de exposição à fatores sabidamente de risco para o VHB nos dois grupos populacionais.

No presente estudo são apresentados resultados de prevalência de infecção pelo VHB em primodoadores de sangue, no Município de Goiânia, $\mathrm{GO}$, e discutidas as implicações metodológicas na interpretação dos estudos seccionais realizados em bancos de sangue para estimativa de prevalência populacional. 


\section{Material e Método}

População de Estudo. Durante o período de outubro de 1988 a fevereiro de 1989 foram entrevistados e testados sorologicamente 1.033 candidatos voluntários a doação de sangue pela primeira vez (primodoadores), atendidos consecutivamente no período matutino em 5 dos 8 bancos de sangue do Município de Goiânia. Foram excluídos indivíduos que se apresentavam em grupo, pertencentes a uma mesma empresa, e militares, devido à dificuldade de se estabelecer a espontaneidade dessas doações.

Cerca de $90 \%$ das doações de sangue do citado município são provenientes dos bancos de sangue estudados e a amostra examinada representou $50 \%$ da demanda total desses bancos, no período de estudo. O tamanho da amostra foi considerado suficiente para estimar uma prevalência esperada para o marcador AgHBs de $0,9 \%$ com uma margem de erro de $2 \%$.

A população rastreada constituiu-se de indivíduos jovens (27 anos, desvio padrão $=9,0$ ), sendo $86,7 \%$ do sexo masculino, $94,4 \%$ residentes em área urbana, $85,9 \% \mathrm{com}$ renda mensal inferior a cinco salários mínimos e $54,1 \%$ com escolaridade até o primeiro grau. O principal motivo da doação foi a solicitação de familiares e amigos $(88,4 \%), 3,8 \%$ para obtenção de exames laboratoriais e $4,2 \%$ foram espontâneas.

Coleta de Dados e Testes Sorológicos. Foram registrados dados de identificação (idade, sexo, residência), renda mensal, grau de instrução e história de icterícia ou hepatite em idade adulta.

Uma amostra de sangue foi coletada para detecção dos marcadores sorológicos AgHBs e antiHBs pela técnica de ensaio imunoenzimático ELI$\mathrm{SA}^{42}$. Os exames foram realizados no Laboratório de Virologia do Departamento de Microbiologia do Instituto de Patologia Tropical e Saúde Pública da Universidade Federal de Goiás (IPTESP/UFG), sendo a preparação dos reagentes biológicos realizada de acordo com Camargo e col. ${ }^{8}$ (1987). Os reagentes foram cedidos pelo Centro de Referência Nacional para Hepatites Virais da Fundação Oswaldo Cruz (FIOCRUZ). A leitura dos resultados foi visual. Os indivíduos que apresentaram positividade ao AgHBs ou anti-HBs foram considerados como já infectados pelo VHB.

Análise Estatística. Como os doadores dos diferentes bancos de sangue não apresentaram diferenças significativas em suas características sóciodemográficas e na prevalência dos marcadores sorológicos estudados ${ }^{31}$, os dados foram analisados em conjunto. Foram calculadas taxas de prevalência para AgHBs e anti-HBs por sexo e idade. Uti- lizou-se o teste de qui-quadrado e o qui-quadrado para tendência ${ }^{30}$ para testar a significância de diferenças entre proporções.

Foram calculados os valores preditivos positivo (VPP) e negativo (VPN) da história pregressa de icterícia e hepatite obtidas pela entrevista, ou seja, a probabilidade de se obter um exame sorológico positivo entre os indivíduos que relataram hepatite e/ou icterícia à entrevista (VPP), ou exames sorológicos negativos na ausência de tal história (VPN).

A sensibilidade da anamnese em detectar infeç̧ão pelo VHB (AgHBs ou anti-HBs positivos) foi calculada pela divisão do número de indivíduos que referiram icterícia ou hepatite e eram soropositivos pelo total de indivíduos soropositivos. A especificidade da anamnese em detectar os não expostos à infeç̧ão pelo VHB foi calculada pela divisão do número dos indivíduos que negaram antecedentes de icterícia ou hepatite e eram soronegativos pelo total de soronegativos (AgHBs e anti-HBs negativos) ${ }^{20}$.

\section{Resultados}

Dos 1.033 doadores $132(12,8 \%)$ foram considerados já infectados pelo VHB (AgHBs ou antiHBs positivo). A prevalência ao anti-HBs foi de $10,9 \%$ e ao AgHBs 1,9\%. Não houve diferença estatisticamente significante entre os sexos masculino e feminino para os marcadores $\mathrm{AgHBs}(2,0 \%$ e $1,5 \%$, respectivamente) e anti-HBs $(11,2 \%$ e $9,5 \%$, respectivamente). Foi observado um aumen-

Tabela 1. Prevalência dos Marcadores Sorológicos da Infecção pelo VHB, AgHBs e anti- AgHBs, por Faixa Etária, em Primodoadores de Sangue, Goiânia-GO, 1988-1989.

\begin{tabular}{|c|c|c|c|c|}
\hline $\begin{array}{l}\text { Faixa etária } \\
\text { (anos) }\end{array}$ & $\begin{array}{c}\text { Primo- } \\
\text { doadores } \\
n\end{array}$ & $\begin{array}{c}\mathrm{AgHBs} \\
n(\%)\end{array}$ & $\begin{array}{c}\text { Anti-HBs } \\
n(\%)\end{array}$ & $\begin{array}{c}\text { AgHBs ou } \\
\text { anti--HBs } \\
n(\%)\end{array}$ \\
\hline $\begin{array}{l}<20 \\
20-29 \\
30-39 \\
40-49 \\
>49\end{array}$ & $\begin{array}{r}162 \\
523 \\
232 \\
78 \\
36\end{array}$ & $\begin{array}{c}0 \\
12(2,3) \\
5(2,2) \\
2(2,6) \\
1(2,8)\end{array}$ & $\begin{array}{r}15(9,3) \\
45(8,6) \\
31(13,4) \\
16(20,5) \\
5(13,9)\end{array}$ & $\begin{array}{r}15(9,3) \\
57(10,9) \\
36(15,5) \\
18(23,1) \\
6(16,7)\end{array}$ \\
\hline Total & $1.033^{* *}$ & $20(1,9)$ & $112(10,9)^{*}$ & $132(12,8)$ \\
\hline
\end{tabular}

to significante da prevalência de anticorpos antiHBs com a idade $\left(\mathrm{X}^{2}\right.$ para tendência $=7,9 \mathrm{p}=$ 0,004) (Tabela 1). 
Tabela 2. Valor Preditivo Positivo (VPP) e Negativo (VPN) da História Pregressa de lctericia ou Hepatite (Anamnese) em Primodoadores de Sangue, Goiânia-Go, 1988-1989.

\begin{tabular}{lcrrr}
\hline \multirow{2}{*}{ Anamnese } & & \multicolumn{2}{c}{ AgHBs ou } & \\
& & Anti-HBs & Total \\
\hline $\begin{array}{l}\text { Ictericia } \\
\text { Ou } \\
\text { Hepatite }\end{array}$ & Sim & 3 & Neg. & \\
\hline Notal & Não & 130 & 881 & 1.011 \\
\hline
\end{tabular}

VPP $3 / 22=13,6 \%$

VPN $881 / 1.011=87,0 \%$

Sensibilidade $3 / 133=2,2 \%$

Especificidade $881 / 900=97,8 \%$

A história de hepatite e/ou icterícia apresentou um valor de predição positivo e uma sensibilidade para detectar indivíduos infectados pelo VHB de $13,6 \%$ e $2,2 \%$, respectivamente. $O$ valor preditivo negativo e a especificidade da história de hepatite ou icterícia foram de $87 \%$ e $97,8 \%$ (Tabela 2).

\section{Discussáo}

No presente estudo a prevalência de $12,8 \%$ de positividade ao AgHBs e/ou anti-HBs indica exposição ao VHB, porém, como o marcador anti-HBs não foi pesquisado, estima-se que $3 \%$ a $5 \%$ dos indivíduos infectados não teriam sido detectados pelo estudo ${ }^{16,24}$. Dessa forma, aproximadamente $83 \%$ da população de primodoadores seria susceptível à infecção pelo VHB.

Em diversas regiões do mundo tem sido verificado uma maior frequiência de AgHBs no sexo masculino $7,18,38$. Além da observação que a duração do marcador sorológico AgHBs pode variar entre homens e mulheres, alguns autores atribuem esses achados a exposições diferenciadas entre os dois grupos. No entanto, alguns estudos populacionais não confirmam esta diferença de prevalência entre sexo ${ }^{26}$. No presente estudo a não detecção de diferenças na prevalência quanto ao sexo, com os marcadores estudados, talvez possa ser explicada por tratar-se de população doadora de sangue supostamente não pertencente a "grupos de risco" onde não se esperaria diferenciais de exposição ao VHB. Por outro lado, o pequeno número de mulheres participantes no estudo não permitiria a detecção de diferenças estatísticas. A razão entre a prevalência dos marcadores anti-HBs $(11,2 \%)$ e AgHBs $(2,0 \%)$ de aproximadamente 6 vezes no sexo masculino, menor do que a habitualmente citada na literatura, talvez possa ser ex- plicada pelas características da população estudada.

O rastreamento sorológico de rotina realizado pelos bancos de sangue de Goiânia nas duas últimas décadas tem registrado prevalências de $\mathrm{AgHB}$ s variando de $0,8 \%$ e $1,3 \% \%^{3,35}$. Entretanto, estas estimativas de prevalência referem-se a percentuais de exames positivos e não indicam, necessariamente, a prevalência entre doadores. Em geral, as estimativas de prevalência referidas pelos bancos de sangue utilizam como denominador 0 total de doações e não o total de doadores. Além do mais, a população doadora é composta por indíviduos que se apresentam à doação pela primeira vez (primodoadores), e por doadores habituais, que já se submeteram a rastreamentos sorológicos prévios, com a provável exclusão dos indivíduos soropositivos. Esses doadores habituais constituem um grupo selecionado do ponto de vista de morbidade, apresentando portanto menor prevalência de infecção ${ }^{27}$. Esta parece ser uma característica importante, pois, em estudo recente, 75\% dos doadores de Goiânia eram habituais e apresentaram uma prevalência para AgHBs de $0,68 \%{ }^{31}$, valor três vezes menor que aquele obtido para os primodoadores no presente estudo $(1,9 \%)$. Esta diferença nas taxas de prevalência de infecção pelo VHB entre primodoadores e doadores habituais tende a ser maior nos locais onde os doadores são informados sistematicamente sobre sua condição de portador, o que limitaria futuras doaçð̋es.

Dessa forma, estimativas de prevalência da infeç̧ão pelo VHB entre primodoadores tendem a se aproximar mais àquela da população geral. Entretanto existem limitações na extrapolação desses resultados.

A primeira consideração a ser feita seria sobre a distribuição etária e sexo da população de primodoadores de sangue. Dos 1.033 primodoadores estudados $95 \%$ tinham entre 20 e 34 anos, sendo $87 \%$ do sexo masculino. No entanto, esta amostra não incluiu indivíduos menores de 15 anos ou maiores de 60 anos que constituíam $40 \%$ da população local (IBGE $\left.{ }^{19}, 1987\right)$.

No intuito de minimizar a tendenciosidade de uma medida de morbidade em doadores de sangue, predominantemente do sexo masculino, vários autores têm utilizado o rastreamento sorológico em gestantes, como "grupo complementar", na tentativa de obter uma melhor estimativa de prevalência para a população adulta em geral ${ }^{1,6}$.

Pelo fato do ato de doação de sangue implicar uma "consciência de saúde", seria esperado um "efeito do doador saudável" em analogia ao "healthy worker effect" descrito para estudo de mortalidade entre trabalhadores ${ }^{34}$. Assim, as estimativas de prevalência observadas em doadores 
subestimariam o nível de infecção populacional em consequiência da seleção prévia. Por ser um ato voluntário, isto também configuraria um importante óbice metodológico pois voluntários podem diferir em muitos aspectos dos não-voluntários ${ }^{28}$. Obviamente estas observações não serão válidas, em amostras que incluam doadores remunerados.

Tem sido enfatizado que dados de prevalência para a infecção pela hepatite $B$, quando baseados na experiência dos serviços de hemoterapia, subestimariam os níveis de infecção porque grupos de alto risco e, conseqüentemente de alta prevalência, estariam sub-representados entre os doadores de sangue ${ }^{22,32}$. Uma tentativa de melhor se aproximar da prevalência populacional "real", implicaria conhecer a frequiência dos grupos de risco dentro da população geral e suas respectivas prevalências de infecção, para o cálculo de uma prevalência ajustada. Considerando-se a similaridade dos mecanismos de transmissão e grupos de risco da infecção pelo VHB e o Vírus da Imunodeficiência Humana (HIV) ${ }^{13,21}$, poder-se-ia utilizar para o VHB o modelo de projeção para a estimativa de prevalência de infecção proposto para o HIV para a população em geral ${ }^{40}$. Nesse modelo a prevalência de infecção obtida em rastreamento sorológico em banco de sangue, considerada como estimativa para a população fora de risco, é ajustada pela prevalência observada nos grupos de risco. Porém, qualquer tentativa de reprodução desse modelo de estimativa populacional no país, fica limitada pela dificuldade de se estabelecer a proporção dos grupos de risco, tais como homossexuais, bissexuais masculinos, usuários de droga e politransfundidos na população geral e suas respectivas prevalências de infecção pelo VHB. Além do mais, não há como comparar e validar os dados de infecção pelo VHB provenientes de bancos de sangue com a magnitude da doença na população, pois a doença não é de notificação compulsória, nem apresenta alta taxa de letalidade que permita analisar $\mathbf{a}$ incidência da doença através das estatísticas de mortalidade. No entanto, a estreita correlação entre a incidência de carcinoma hepatocelular e prevalência de AgHBs em nível populacional tem sido utilizada para sinalizar áreas de alta endemicidade de infecção $0^{4,39}$.

As prevalências de $1,9 \%$ para AgHBs e $10,9 \%$ para anti-HBs, observada nos primodoadores de sangue, caracterizam Goiânia como baixa a intermediária endemicidade ${ }^{44}$, a semelhança do que foi visto no Rio de Janeiro ${ }^{43}$. A variação nas taxas de prevalência por faixa etária configura riscos diferenciados de infecção, na dependência do nível endêmico de cada região, $0^{2,29}$. Para os primodoadores de Goiânia, observou-se uma tendência crescente na prevalência de anticorpos com a idade, indican- do uma maior oportunidade de exposição, provavelmente via contato sexual. Em áreas de alta endemicidade, como na região Norte do Brasil, prevalências de $19,2 \%$ para AgHBs foram detectadas em crianças menores de 15 anos, sendo que na faixa etária de 10-14 anos $75 \%$ da população da área já apresentava evidência sorológica de infeç̧ão ao $V H^{5}$. Nessa situação o rastreamento em população adulta, como os doadores de sangue, não expressaria a distribuição da infecção na população umà vez que a população infantil estaria excluída.

A avaliação da anamnese para triagem da infecção pelo VHB em bancos de sangue indicou baixa sensibilidade e baixo valor de predição para exposição ao VHB. Na verdade este fato era esperado, tendo-se em vista a história natural da infecção pelo VHB, com elevados percentuais de infeç̧ão subclínica ${ }^{4}$.

A comparação entre resultados de diferentes rastreamentos sorológicos deve levar em consideração as diferenças em sensibilidade e especificidade das técnicas laboratoriais empregadas. Mesmo técnicas altamente sensíveis como a ELISA apresentam valores de predição variáveis de acordo com a prevalência da infecção na população estudada ${ }^{20}$.

Embora os resultados de rastreamentos realizados em doadores de sangue não possam a rigor ser considerados indicadores de infecção para o VHB em nível populacional, a padronização na triagem e registro dos candidatos, e nas técnicas laborato. riais utilizadas, tomariam os bancos de sangue locais privilegiados para o estudo da tendência secular dessa infecção em indivíduos adultos, com finalidade de vigilância epidemiológica.

\section{Agradecimentos}

Ao Conselho Nacional de Desenvolvimento Científico e Tecnológico $(\mathrm{CNPq})$ e Secretaria de Ciência e Tecnologia do Ministério da Saúde pelo apoio financeiro; à Dra. Clara F. T. Yoshida, do Centro de Referência de Hepatites Virais da FIOCRUZ, pelo apoio laboratorial; e ao Dr. César Leite de Santana, diretor do Hemocentro de Goiás.

MARTELLI, C. M. T. et al. [Methological issues in the interpretation of serologic screening for hepatitis $\mathrm{B}$ virus among blood donor's]. Rev. Saúde públ., S.Paulo, 25: 11-6, 1991. Between October 1988 and February 1989 , 1,033 voluntary first-time blood donors were screened for hepatitis B infection in five blood banks in Goiânia, Central Brazil. The survey was part of a major study designed to estimate seroprevalence of $\mathrm{HBsAg}$ 
and anti-HBs and to discuss methodological issues related to prevalence estimation based on data from blood banks. Donors were interviewed and blood samples were collected and tested for $\mathrm{HBsAg}$ and anti-HBs by ELISA tests. Prevalences of $1.9 \%$ and $10.9 \%$ were obtained for $\mathrm{HBsAg}$ and anti-HBs, respectively, and no statistical difference was found between the sexes. Prevalence of anti-HBs increased with age $\left(X^{2}\right.$ for trend $=7.9 \mathrm{p}=0.004$ ). The positive predictive value and sensitivity of history of jaundice or hepatitis reported in the interview in detecting seropositives were $13.6 \%$ and $2.2 \%$, respectively. The methodological issues, including internal and external validity of HBV prevalence estimated among blood donors are discussed. The potential usefulness of blood banks as a source of morbidity information for surveillance for Hepatitis B virus infection is stressed.

Keywords: Hepatitis B, diagnosis. Serodiagnosis. Prevalence studies. Blood donors.

\section{Referências Bibliográficas}

1. ABIODUN, P.O.; IHONGUE, J.C.; UBARU, R.; KRAULEDAT, P. HBsAG carrier rate among the adult population in Benin City, Nigeria (ELISA Method). Publ. Hlth, 100: 362-7, 1986.

2. ABUZWAIDA, A.R.N.; SIDONI, M.; YOSHIDA, C.F.T.; SCHATZMAYR, H.G. Seroepidemiology on Hepatitis $B$ in two urban communities of Rio de Janeiro, Brazil. Rev. Inst. Med. trop. S. Paulo, 29: 219-33, 1987.

3. ANDRADE, A.L.S.S.; MARTELLI, C.M.T.; PINHERIO, E.D.; SANTANA, C.L.; BORGES, F.P.; ZICKER. F. Rastreamento sorologico para doenças infecciosas em banco de sangue como indicador de morbidade populacional. Rev. Saúde públ., S. Paulo, 23: 20-5, 1989.

4. BEASLEY, R.P. Hepatitis $B$ virus as the etiologic agent in hepatocellular carcinoma: epidemiologic considerations. Hepatology, 2: 215-65, 1982.

5. BENSABATH, G.; HADLER, S.C.; SOARES, M.C.P.; FIELDS, H.; MAYNARD, J. Caracteristicas serologicas y epidemiologicas de la hepatitis virica aguda. Bol. Ofic. sanit.panamer., 103: 351-62, 1987.

6. BILE, K.M.; ADEN, A.; LINDBERG, G.; NILSSON, L.; LIDMAN, K.; NORDER, H.; MAGNIUS, L. Epidemiology of Heptatitis B in Somalia: inference from a cross-sectional survey of serological markers. Trans. roy. Soc. trop. Med. Hyg., 81: 824-8, 1987.

7. BLUMBERG, B.S.; SUNICK, A.I,; LONDON, W.J. Sex distribution of Australia antigen. Arch. intern. Med., 130: 227-31, 1972.

8. CAMARGO, I.F.; GASPAR, A.M.C.; YOSHIDA, C.F.T. Comparative Elisa reagents for detection of hepatitis $B$ surface antigen (HBsAG). Mem. Inst. Oswaldo Cruz, 82: $181-7,1987$.

9. CARRILHO, F.J. \& SILVA, L.C. Epidemiologia. In: Silva L.C., ed. Hepatites agudas e crônicas. São Paulo, Sarvier, 1986. p. 47-69.

10. CARRILHO, F.J.; BALDY, J.L. da S.; TAKATA, P.K.; ADUM, S.; ZEITUNE, J.M.R. Prevalência e estudo de portadores do antígeno de superfície do vírus da hepatite B (AgHBs) em Londrina, Paraná. GED: Gastroenterol. Endosc. dig., 3(1): 13-20, 1984.

11. CENTERS FOR DISEASE CONTROL. Changing pattems of groups at high risk for hepatitis $B$ in The United States. Morb. Mort. wkly Rep., 37: 429-37, 1988.
12. CENTERS FOR DISEASE CONTROL. Prevention of perinatal transmission of Hepatitis $B$ virus: prenatal screening of all pregnant women for Hepatitis B surface antigen. Morb. Mort. wkly Rep., 37: 341-6, 351, 1988.

13. COCK, K.M.; NILAND, J.C.; LU, H.P.; RAHIMIAN, A.; EDWARDS, V.; SHRIVER, K.; GOVINDARAJAN, S.; REDEKER, A.G. Experience with Human Immunodeficiency Vinus infection in patients with Hepatitis $B$ Virus and Hepatitis Delta Virus infections in Los Angeles, 1977-1985. AmerJ. Epidem., 127: 1250.9, 1988.

14. DECKER, M.D; VAUGHN, W.K.; BRODIE, J.S.; HUTCHESON, R.H.; SCHAFFNER, W. Seroepidemiology of Hepatitis B in Tenesse prisioners. J. infect. Dis., 150: 450-9, 1984

15. DIENSTAG, J. L. \& RYAN, D. M. Occupational exposure to Hepatitis B Virus in hospital personnel: infection or immunization? Amer. J. Epidem. 115: 26-39, 1982.

16. EDWARDS, M.S. Hepatitis B serology help in interpretation. Pediat. Clin. North Amer, 35: 503-15, 1988.

17. FIGUEIREDO, J.F.C.; MYSES NETO, M.; GOMES, U.A.; FERRAZ, A.S.; BATISTA, M.E.P.N.; GASPAR, A.M.C.; YOSHIDA, C.M.T. Hepatitis B virus infection in hemodialysis units: clinical features, epidemiological markers and general control measures. Braz. J. med. biol. Res., 19: 735-42, 1986.

18. FOX, E.; ABBATTE, E.A.; SALAH, S.; CONSTANTDNE, N.T.; WASSEF, H.H. WOODY, J.N. Viral hepatitis markers in Djibouti: an epidemiological survey. Trans, roy. Soc. trop. Med. Hyg., 82: 750-2, 1988.

19. FUNDAÇĀO IBGE. Anuário Estatístico do Brasil. Rio de Janeiro, 1987/1988.

20. GALEN, R.S. \& GAMBINO, S.R. Beyond normality: the predictive value and efficiency of medical diagnoses. New York, John Wiley \& Sons, 1975.

21. GARIBALDI, R.A. Transmission of Heptatitis $B$ and AIDS. Infect. Control, 7(Suppl.): 132-4, 1986.

22. GUST, I.D. The epidemiology of viral hepatitis. In: Vyas, G.N.; Dienstag, J.L.; Hoofnagle, J.H., eds. Viral hepatitis and liver diseases. New York, Grune \& Stratton, 1984. p. 415-21.

23. HADLER, S.C.; FAY, O.H.; PINHEIRO, F; MAYNARD, J.E. La hepatitis en las Americas: informe del grupo colaborador de la OPS. Bol. Ofic. sanit. panamer. 103: 185-208, 1987.

24. HOOFNAGLE, J.H. Serologic markers of hepatitis B virus infection. Ann Rev Med., 32: 1-11, 1981.

25. HYAMS, K.C.; PALINKAS, L.A.; BURR, R.G. Viral hepatitis in the U.S. Navy, 1975-1984. Amer. J. Epidem., 130: 319-26, 1989.

26. KASHIWAGI, S.; HAYASHI, J.; IKEMATSU, H.; NOMURA, H.; KUSABA, T; SHINGU, T.; HAYASHIDA, K.; KAJ, M. An epidemiologic study of hepatitis B virus in Okinawa and Kyushu, Japan. Amer. J. Epidem., 118: 787-98, 1983.

27. KAY, L.A. Retention of regular blood donors. Vox Sang., 56: 204, 1989.

28. KLENBAUM, D.G.; KUPPER, L.L.; MORGENSTERN, H. Epidemiologic research. Belmont, Cal., Lifetime Leaming Publ., 1982.

29. LYRA, L.G.; DAMASCENO, A.P; COTRIM, P.; MOTA, E.; SILVA, L. Prevalence of antibody to hepatitis $B$ virus in an urban population of Northeast Brazil. Rev. Inst. Med. trop. S.Paulo, 28: 406-9, 1986.

30. MANTEL, N. Chi-square tests with one degree of freedom; extension of the Mantel-Haenszel procedure. $J$. Amer. statist. Ass., 58: 690-700, 1963. 
31. MARTELLI, C.M.T. Estudo epidemiológico da infecção pelo vírus da Hepatite $B$ em primodoadores de sangue de Goiânia. Goiânia, 1989. [Dissertação de Mestrado - Instituto de Patologia Tropical e Saúde Pública da UFG].

32. MARTELLI, C.M.T.; ANDRADE, A.L.S.S.; CARDOSO, D.D.P.; SOUSA, L.C.S.; SILVA, S.A.; SOUZA M.A.; ZICKER, F. Soroprevalência e fatores de risco para a infecção pelo víns da hepatite $B$ em prisioneiros e primodoadores de sangue. Rev. Saúde públ., S. Paulo, 24: 270-6, 1990.

33. MAZZUR, S.; NATH, N.; FANG, C.; BASTIAANS, M.J.; MOLINARIS, J.L.; BALCASER, M.; BEKER, G.S.; BRUNINGS, E.A.; CAMERON, A.R.E.; FARREL, V.; FAY, O.H.; LABRADOR-GONZALEZ, G.; GUTIERREZ, D.A.; JARAMIILO, T.C.; KATZ, R.; LEME-LOPEZ, M.B.; LEVY-KOENIG, E.; MORALES AYALA, F.; RODRIGUEZ AMAYA, J.; RODRIGUEZ-MOYADO, H.; TORRES, R.A. de; VELASCO, M. Distribucion de marcadores de virus de hepatitis B (VHB) en donatores de sangre de 13 paises del hemisferio ocidental: actas del Taller Latinoemaricano de Cruz Roja sobre Hepatitis B. Bol. Ofic. sanit. panamer., 89: 239-47, 1980.

34. MCMICHAEL, A.J. Standardized mortality ratios and the healthy worker effect: scratching beneath the surface. J. occup. Med., 18: 165-8, 1976.

35. ROSA, H.; LEMOS, Z.P.; MAGALHÃES, A.F.N.; CARDOSO, V.M. HAA, imunoglobulinas e autoanticorpos em retardados mentais, doadores de sangue e hepatite viral aguda. In: Congresso Brasileiro de Gastroenterologia, 24․, Petrópolis, 1974. Anais. Petropolis, 1974. p. 175.

36. SCHEUTZ, F.; MELBYE, M.; ESTEBAN, J.I.; ALDERSHVILE, J.; EBBESEN, P.; ALTER, H.J. Hepatitis $B$ virus infection in Danish dentist: a case control and follow up study. Amer.J.Epidem ., 128: 190-6, 1988.
37. SCHREEDER, M.T.; THOMPSON, S.E.; HADLER,S.C.; BERQUIST, K.R.; ZAIDI, A.; MAYNARD, J.E.; OSTROW, D.; JUDSON, F.N.; BRAFF, E.H.; NYLUND, T.;MORRE JR., J.N.; GARDNER, P.; DOTO, I.L.; REYNOLDS, G. Hepatitis B in homossexual men: prevalence of infection and factor related to transmission. J. infect. Dis., 146: 7-15, 1982.

38. SHERLOCK, S. The natural history of hepatitis B. Postgrad. med. J., 63(2): 7-11, 1987.

39. SILVEIRA, A.C. \& SILVEIRA, C.M. Associação entre hepatite B e carcinoma hepatocelular no Estado do Amazonas, Brasil: dados de mortalidade - 1979/ 1984. Rev. Soc. bras. Med. trop., 21(Supl.): 42, 1988.

40. SIVAK, S.L. \& WORMSER, G.P. How commom is HTLV-III infection in the United States? New Engl.J. Med., 313: 1352, 1985.

41. SZMUNESS, W.; MUCH, I.; PRINCE, A.M.; HOOFNAGLE, J.H.; CHERUBIN, C.E.; HARLEY, E.J.; BLOCK, G.H. On the role of sexual behavior in the spread of hepatitis B infection. Ann. intern. Med., 83: 489-95, 1975.

42. VOLLER, A.; BARTETT, A.; BIDWEI, D.E. Enzyme immunoassays with special reference to ELISA techniques. J. clin. Pathol.; 31: 507-20, 1978.

43. YOSHIDA, C.F.T.; NOGUEIRA, R.M.R.; MERCADANTE, L.A.C.; PINHÃO, A.T.; SCHATZMAYR, H.G. Seroepidemiological survey of hepatitis $A$ and $B$ cytomegalovirus and herpes simplex Type 2 in prime blood donors from Rio de Janeiro, Brazil. Rev. Microbiol., 18: 5-11, 1987.

44. ZUCKERMAN, A.J. Controversies in immunization against hepatitis B. Hepatology, 5: 1227-30, 1985.

Recebido para publicação em 19/1/1990 Reapresentado em 2/10/1990 Aprovado para publicaçäo em 4/10/1990 